\title{
Limited genomic divergence between intraspecific forms of Culex pipiens under different ecological pressures
}

\author{
Bruno Gomes ${ }^{1,2^{*}}$ D, Craig S. Wilding ${ }^{2,3}$, David Weetman ${ }^{2}$, Carla A. Sousa ${ }^{1}$, Maria T. Novo ${ }^{1}$, Harry M. Savage ${ }^{4}$ \\ António P. G. Almeida', João Pinto ${ }^{1}$ and Martin J. Donnelly ${ }^{2}$
}

\begin{abstract}
Background: Divergent selection can be a major driver of ecological speciation. In insects of medical importance, understanding the speciation process is both of academic interest and public health importance. In the West Nile virus vector Culex pipiens, intraspecific pipiens and molestus forms vary in ecological and physiological traits. Populations of each form appear to share recent common ancestry but patterns of genetic differentiation across the genome remain unknown. Here, we undertook an AFLP genome scan on samples collected from both sympatric and allopatric populations from Europe and the USA to quantify the extent of genomic differentiation between the two forms.

Results: The forms were clearly differentiated but each exhibited major population sub-structuring between continents. Divergence between pipiens and molestus forms from USA was higher than in both inter- and intra-continental comparisons with European samples. The proportion of outlier loci between pipiens and molestus $(\approx 3 \%)$ was low but consistent in both continents, and similar to those observed between sibling species of other mosquito species which exhibit contemporary gene flow. Only two of the outlier loci were shared between inter-form comparisons made within Europe and USA.
\end{abstract}

Conclusion: This study supports the molestus and pipiens status as distinct evolutionary entities with low genomic divergence. The low number of shared divergent loci between continents suggests a relatively limited number of genomic regions determining key typological traits likely to be driving incipient speciation and/or adaptation of molestus to anthropogenic habitats.

\section{Background}

Divergent selection is a major driving force in speciation models involving taxa with overlapping geographic distributions, either during sympatric speciation or via reinforcement of isolation between allopatric incipient species after secondary contact $[1,2]$. The capacity for divergent selection to promote reproductive isolation among populations depends on the strength of selection, the number of traits upon which selection is acting and the rates of realised gene flow [3]. Initially, Wu [4]

\footnotetext{
* Correspondence: Bruno.GomesdaSilva@lstmed.ac.uk

${ }^{1}$ Global Health \& Tropical Medicine, Instituto de Higiene e Medicina Tropical, Universidade Nova de Lisboa, Rua da Junqueira 100, 1349-008 Lisbon,

Portugal

${ }^{2}$ Department of Vector Biology, Liverpool School of Tropical Medicine,

Pembroke Place, Liverpool L3 5QA, UK

Full list of author information is available at the end of the article
}

proposed that only strong selection concentrated on a few traits may overcome substantial gene flow, at least for those specific genomic regions which initiate sympatric speciation. However, recent studies have shown much wider divergence across numerous genomic regions between closely related insect ecotypes [5-7].

In insects of medical importance, the speciation process may also have a public health dimension. Culex pipiens sensu stricto is a widespread mosquito species with an important medical and veterinary impact owing to its role in the transmission of arthropod-borne viruses (arboviruses) such as the potentially-fatal zoonotic West Nile virus [8]. Culex pipiens s.s. is comprised of two distinct forms, denoted pipiens and molestus, which are morphologically indistinguishable but exhibit behavioural and physiological differences that are likely to 
impact pathogen-transmission. The molestus form is differentiated from pipiens by four key ecological/physiological characteristics: autogeny (the capacity to lay eggs without taking a blood meal), stenogamy (the capacity to mate in confined spaces), homodynamy (a continuous life cycle without diapause), and mammophily (a preference to feed on mammals, including humans) $[9,10]$.

In southern European/Mediterranean regions, the two $C x$. pipiens s.s. forms are sympatric in aboveground habitats, but in northern regions of Europe, Russia and the USA, molestus and pipiens segregate into underground and aboveground habitats, respectively [11-13]. A continuous life cycle may be a limitation for surviving in colder climates which may restrain the habitat choice of molestus, while autogeny and stenogamy are important traits for survival in confined underground habitats with restricted access to blood meals. Genomic regions associated with these differentiated traits are currently unknown, as is the degree of ecologically-driven genomic divergence between the forms.

Populations with mixed characteristics between molestus and pipiens have been found in southern European regions [13-15] where inter-form gene flow has been detected, resulting in a pattern of asymmetric introgression from molestus into pipiens [13, 16]. Moreover, an unusual biting preference for birds has been described in the molestus form in southern Europe [17]. Populations with mixed characteristics were also found in USA [18].

Two hypotheses have been proposed for the origin of molestus and pipiens forms. One that the molestus form is polyphyletic; derived from the pipiens form through multiple independent adaptations to underground anthropogenic habitats [11]. The second hypothesis considers molestus as an evolutionarily independent entity from southern latitudes, which has secondarily colonized northern underground habitats [12]. Microsatellitebased studies showed common ancestry of geographically distinct populations of molestus, supporting its status as a single evolutionary entity [12]. However, these studies did not compare aboveground European molestus (in sympatry with pipiens form) and American underground molestus with other geographic populations of this form.

In this study, we performed an AFLP-based genome scan on geographically-distinct $C x$. pipiens s.s. samples. The main goals of this study were: i) to determine if European and American populations of each form present similar genetic backgrounds; ii) to infer the divergence between molestus and pipiens forms by $F_{S T}$ estimates; and iii) to quantify outlier rates in inter-form comparisons. Our results provide an insight into how the genetic background of pipiens and molestus forms varies based on their geography and population characteristics (natural/colony populations). This information is crucial for understanding the impacts of habitat adaptation and ecological speciation within this species.

\section{Results}

\section{Dominant markers and error rates}

A total of 894 dominant markers were obtained from 12 primer combinations used in the selective amplification (see Additional file 1: Tables S1 and S2). The markers obtained by the primer combinations EcoRI-ACG/MseICGA (Mix1D3) and EcoRI-ACG/MseI-ACC (Mix3D3) yielded high proportions of mismatches between replicates (12.50 and $19.58 \%$, respectively) and were removed prior to subsequent analysis. The proportion of mismatches from the remaining 810 dominant markers varied between 0.00 and $1.02 \%$ (mean: $0.33 \%$ ). Error rates for these 10 primer combinations averaged 1.41 and $0.04 \%$ for the probabilities calculated by AFLPscore [19] of mis-scoring a peak as absent if present, and vice versa. Error rates for each primer combination are detailed in Additional file 1: Table S2.

The dataset showed an average of 81 loci per primercombination with only two combinations yielding more than 100 loci (EcoRI-CTC/MseI-CAA - Mix2D4, EcoRI-CTC/MseI-AGT - Mix4D4; Table S2). The 810 loci presented a balanced distribution among fragment size groups: 172 loci $(21.2 \%)$ exhibited small fragment sizes $(<125 \mathrm{bp})$ and 233 loci $(28.8 \%)$ largest fragment size ( $>299 \mathrm{bp}$ ), with all remaining fragments 125 $299 \mathrm{bp}$. This dataset complies with the technical recommendation to avoid an imbalanced number of loci per primer-combination and an excessive proportion of loci of small fragment size, thus reducing potential for peak size homoplasy [20].

\section{Population clustering analysis}

STRUCTURE [21] analysis of all 327 female mosquitoes analysed for the 810 loci indicated an optimum of two clusters (see Additional file 1: Fig. S1). Division into the two clusters closely matched the previous formidentification used to select the mosquito samples (full description in Methods, Mosquito samples). However, eight individuals previously identified as molestus (five from Sandim and three from Comporta) presented an individual assignment inferior to 0.50 for this cluster (Fig. 1a). Principal component analysis (PCA) performed by GENALEX 6.41 [22] confirmed the division between the two forms and the placement of the eight previouslyidentified molestus females closer to pipiens form individuals (Fig. 2). These eight individuals were excluded from the subsequent analysis owing to the conflicting classification between the AFLP data and the other identification methods.

The average probability of membership $\left(A v \cdot q_{i}\right)$ obtained by the STRUCTURE varied among geographic 


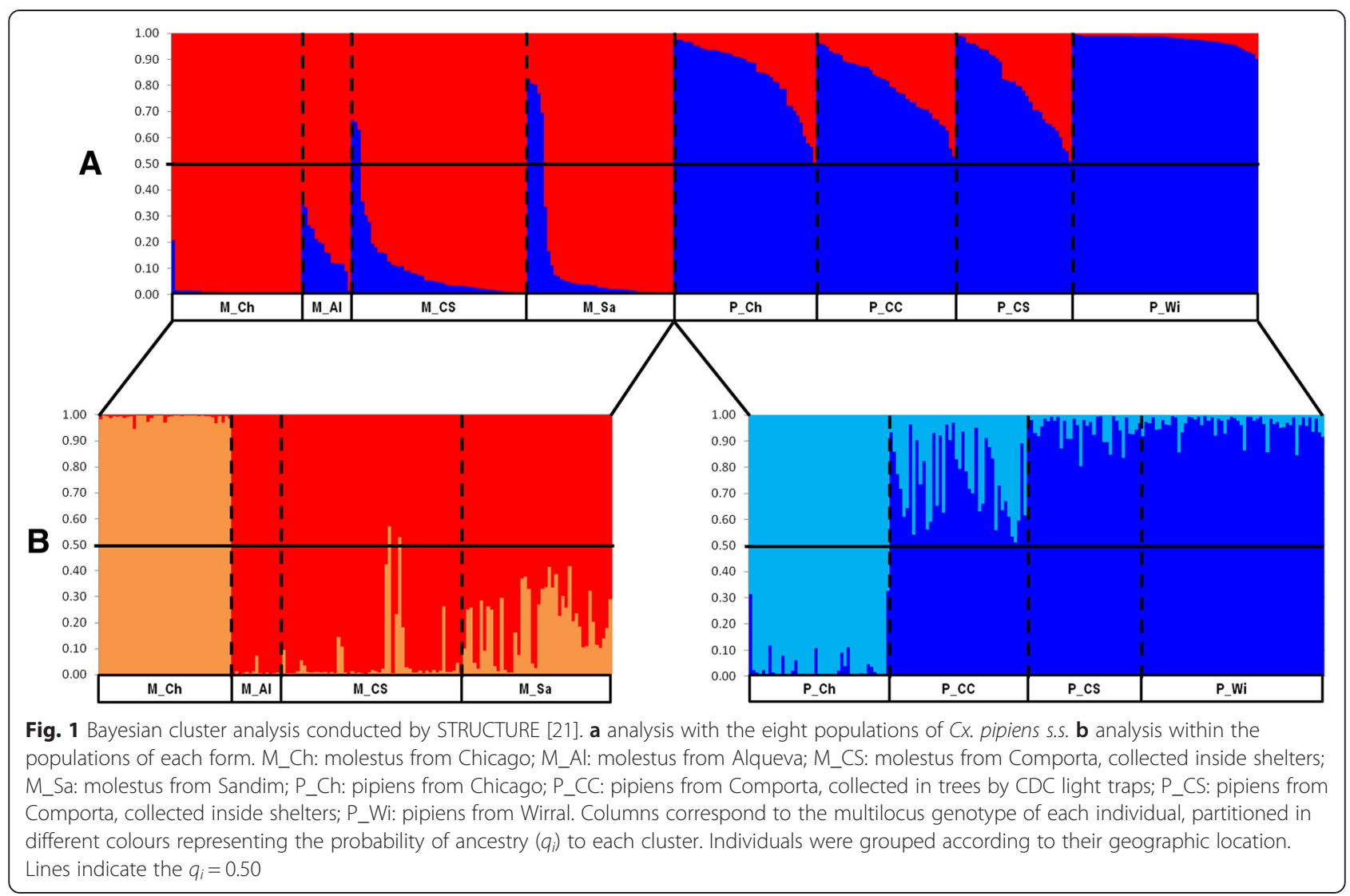

samples. In pipiens samples, the UK sample showed a higher $A v \cdot q_{i}$ (0.976) than the Chicago (USA) sample (0.850) and Comporta (PT) samples (0.779-0.800). In the molestus form, Portuguese samples displayed lower $A v \cdot q_{i}(0.820-0.893)$ than the Chicago (USA) sample (0.985). The consistently lower $A v \cdot q_{i}$ in Portuguese samples suggests a higher degree of admixture than in the other geographic samples.

Clustering analysis was also performed within each form separately; both analyses indicated a division into two clusters, which split Chicago samples from European samples, within each form (Fig. 1b and Additional file 1: Fig. S1). PCA supported the geographic (continental) division within molestus (Fig. 2a) and pipiens (Fig. 2b), with European samples of each form comprising a single group but the samples from Chicago (USA) separated from all the other samples. A neighbour-joining tree based on $F_{S T}$ values supported the division between the forms and also a high differentiation between the European and American samples, especially in the molestus form (Fig. 3).

AMOVA [23] apportioned $11.7 \%$ of the molecular variance among populations, and only $5.9 \%$ of the variation between the two forms. When the analysis was repeated using European samples alone, the molecular variance among populations fell to $5.6 \%$, whereas between forms increased to $8.4 \%$.
Diversity estimates were calculated for each population by the AFLP-SURV [24] (see Additional file 1: Table S3). No significant differences were found between pipiens and molestus forms in number of polymorphic loci (Loc_P: $X^{2}=2.09 ;$ d.f. $=2 ; P=0.35$ ), and the proportion of polymorphic loci at the $5 \%$ level (PLP: $\chi^{2}=0.25$; d.f. $=2 ; P=0.88$; Additional file 1: Table S3).

\section{Population differentiation}

The $F_{S T}$ estimates (i.e., mean, median, maximum and three percentiles) used to map divergence among four subsamples presented in our data set (i.e., pipiens and molestus from Europe and USA, respectively) are shown in Table 1 and the Additional file 1: Tables S4 and S5. Comparative pairwise analyses were performed using mean/median and maximum values because those for lower percentiles and the minimum values were negative values of $F_{S T}$.

For almost every measurement of $F_{S T}$ intra-form comparisons were consistently higher between USA and Europe than among populations within Europe (pipiens: $\approx 2.3 \times$ higher on average; molestus: $\approx 2.8 \times$ higher on average). Between pipiens and molestus forms, $F_{S T}$ values were higher between the USA samples than in any intercontinental comparison between Europe and USA $(\approx 1.3 \times)$. Inter-form comparisons between European 


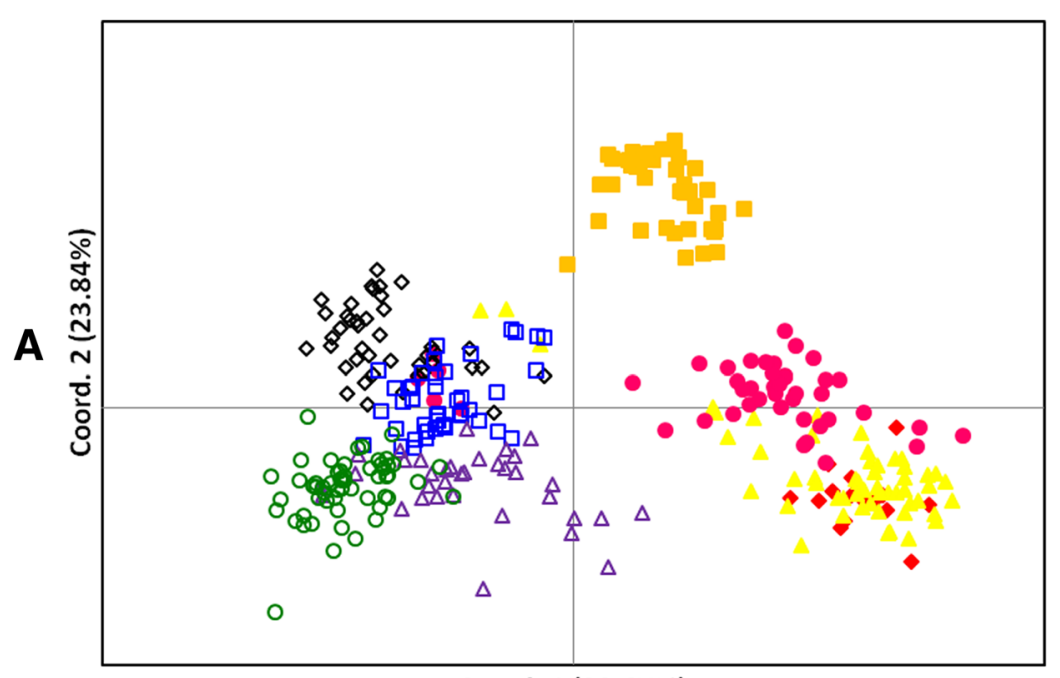

M_Ch

- M_Al

M_CS

- M_Sa

$\diamond P_{-}$Ch

$\square$ P_CC

$\triangle P_{-} C S$

$\circ P_{-}$Wi

Coord. 1 (29.65\%)

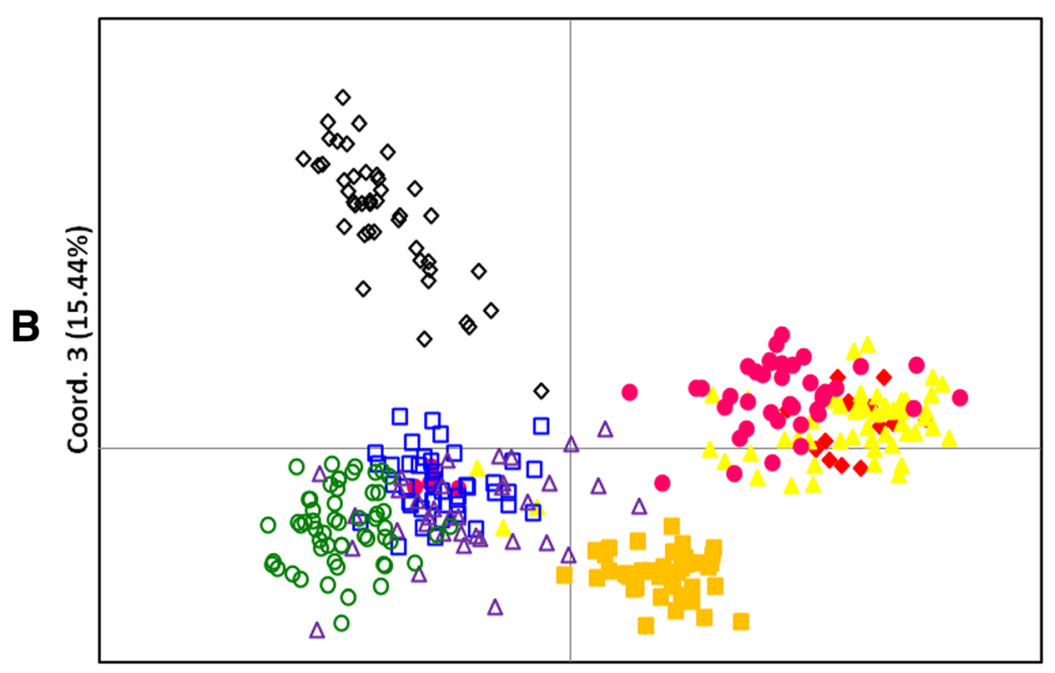

- M_Ch

- M_Al

M_CS

- M_Sa

$\diamond P_{-}$Ch

$\square$ P_CC

$\triangle P_{-} C S$

OP_Wi

Coord. $1(29.65 \%)$

Fig. 2 Principal Coordinates Analysis of the eight CX. pipiens s.5. samples conducted by GENALEX 6.41 [22]. a two-dimensional plots of principal coordinates 1 and 2; $\mathbf{b}$ two-dimensional plots of principal coordinates 1 and 3. M_Ch: molestus from Chicago; M_Al: molestus from Alqueva; M_CS: molestus from Comporta, collected inside shelters; M_Sa: molestus from Sandim; P_Ch: pipiens from Chicago; P_CC: pipiens from Comporta, collected in trees by CDC light traps; P_CS: pipiens from Comporta, collected inside shelters; P_Wi: pipiens from Wirral.

Coord: coordinate (percentage of variation explained by each coordinate)

samples yielded lower $F_{S T}$ values than USA $(\approx 1.9 \times)$ and intercontinental comparisons $(\approx 1.5 \times)$.

In 5 out of 6 comparisons involving the USA molestus sample higher $F_{S T}$ values were found in intra-form comparisons than in inter-form comparisons with European samples $(\approx 1.2 \times$; Table 1$)$. The high divergence found between intercontinental samples of molestus is illustrated in the neighbour-joining tree (Fig. 3).

\section{Detecting outlier loci}

Due to the marked genetic structure between American and European samples, the detection of outlier loci was performed separately for each continent. Results of the outlier analysis performed using three different approaches among all the European population samples $(N=6)$ and within each form in Europe $(N=3)$ are shown in Fig. 4 and Additional file 1: Fig. S2.

In Europe, a total of 25 loci (3.1\%) were scored as outliers across the three methods performed by BAYESCAN $2.1[25,26]$ and MCHEZA [27]. However, this number varied among the methods and only six out of the 25 outlier loci were detected consistently by all methods: Mix1D2_022, Mix3D4_041, Mix4D2_004, Mix4D3_016, Mix4D4_011, and Mix4D4_037 (Fig. 5).

MCHEZA detected 13 (1.6\%) loci as outliers between the molestus and pipiens samples from Chicago (USA) 


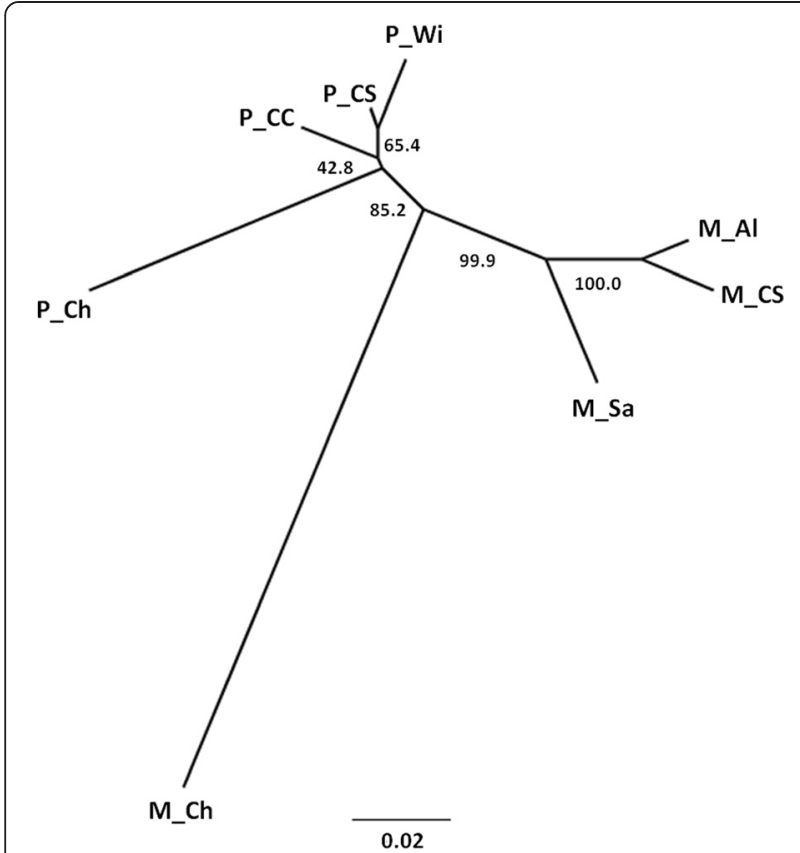

Fig. 3 Unrooted Neighbour-joining tree based on $F_{S T}$ values. Bootstrap (\%) support of each branch is given. M_Ch: molestus from Chicago; M_Al: molestus from Alqueva; M_CS: molestus from Comporta, collected inside shelters; M_Sa: molestus from Sandim; P_Ch: pipiens from Chicago; P_CC: pipiens from Comporta, collected in trees by CDC light traps; P_CS: pipiens from Comporta, collected inside shelters; P_Wi: pipiens from Wirral

but neither of the approaches implemented by BAYESCAN detected any outliers (Fig. 5). Of the 36 total outlier loci found either in Europe or in USA, only two (0.25 \%), Mix3D4_041 (outlier by all methods in Europe) and Mix4D4_027 (outlier only by MCHEZA), were found consistently across both continents (see Additional file 1: Table S6).

However, USA samples exhibited only half the number of scored polymorphic loci $(N=406)$ compared with European samples. In fact, for six of the within-Europe outliers (Mix1D4_006, Mix1D4_063, Mix2D2_039, Mix4D2_023, M4D2_049, Mix4D3_044) no positive band was detected in the USA samples, precluding its inclusion in the USA analysis. This drastic reduction of polymorphic loci in the USA data set led to a higher proportion of small-sized loci $(33.7 \%)$ that significantly changed the loci distribution among fragment size groups when compared with the original $\left(\chi^{2}=45.83\right.$, d.f. $=3, P<0.0001$; see Additional file 1: Table S7).

\section{Discussion}

In this study, a rigorously quality-controlled AFLP procedure was applied to understand the nature of differentiation within and between pipiens and molestus forms of Culex pipiens from two continents. This genomewide AFLP scan provides additional evidence supporting the hypothesis that molestus and pipiens forms correspond to evolutionarily distinct entities [12]. Independent of geographic origin, molestus samples clustered together and were genetically distinct from pipiens samples highlighting a common ancestry between European and USA molestus populations. This result was consistent in all analyses of population structure conducted. In addition to the molestus/pipiens partitioning, population sub-structuring was found between continents within each form.

Inter-continental differentiation was higher within the molestus than the pipiens form, possibly due to two factors. 1) Colonization of an underground habitat by the USA molestus population studied (which was a sealed habitat blocking contact with other populations [28]). This may have increased genetic drift associated with founder effects/bottlenecks. High differentiation has also been observed between natural populations of molestus in Chicago and New York [29]. 2) Laboratory colonization of the samples analysed and their maintenance for 2 years is very likely to have inflated differentiation compared to that expected among equivalent natural source

Table 1 Divergence estimates of $F_{S T}$ pairwise sample analysis per locus

\begin{tabular}{|c|c|c|c|c|c|c|c|c|c|c|c|c|}
\hline & \multicolumn{3}{|c|}{ Molestus } & \multicolumn{3}{|c|}{ Pipiens } & \multicolumn{6}{|c|}{ Molestus vs. pipiens } \\
\hline & All & EU & EUvsUSA & All & EU & EUvsUSA & All & EU & USA & EUvsUSA & EUvsM_Ch & EUvsP_Ch \\
\hline Max & 0.940 & 0.597 & 0.940 & 0.793 & 0.793 & 0.750 & 0.942 & 0.806 & 0.942 & 0.938 & 0.938 & 0.837 \\
\hline Per 99 & 0.595 & 0.346 & 0.718 & 0.320 & 0.204 & 0.355 & 0.511 & 0.433 & 0.798 & 0.596 & 0.648 & 0.553 \\
\hline Per 95 & 0.261 & 0.159 & 0.386 & 0.137 & 0.083 & 0.177 & 0.241 & 0.201 & 0.357 & 0.288 & 0.261 & 0.320 \\
\hline Per 75 & 0.065 & 0.038 & 0.092 & 0.032 & 0.015 & 0.054 & 0.060 & 0.054 & 0.121 & 0.076 & 0.069 & 0.096 \\
\hline Median & 0.008 & 0.004 & 0.021 & 0.005 & -0.001 & 0.013 & 0.014 & 0.012 & 0.029 & 0.022 & 0.025 & 0.021 \\
\hline Mean & 0.052 & 0.028 & 0.080 & 0.027 & 0.014 & 0.041 & 0.051 & 0.041 & 0.091 & 0.064 & 0.060 & 0.069 \\
\hline N & 3,038 & 1,621 & 1,417 & 4,401 & 2,296 & 2,105 & 10,492 & 6,658 & 404 & 3,711 & 2,075 & 1,636 \\
\hline
\end{tabular}

All: within all pairwise comparison; EU: pairwise comparison within European samples; USA: pairwise comparison within USA samples; EUvsUSA: pairwise comparison between European and USA samples; EUvsM_Ch: pairwise comparison between European samples and molestus from Chicago; EUvsP_Ch: pairwise comparison between European samples and pipiens from Chicago; Max: maximum $F_{S T}$ value; Per X: percentile X\% of the $F_{S T}$ values distribution; $N$ : total number of pairwise comparison 

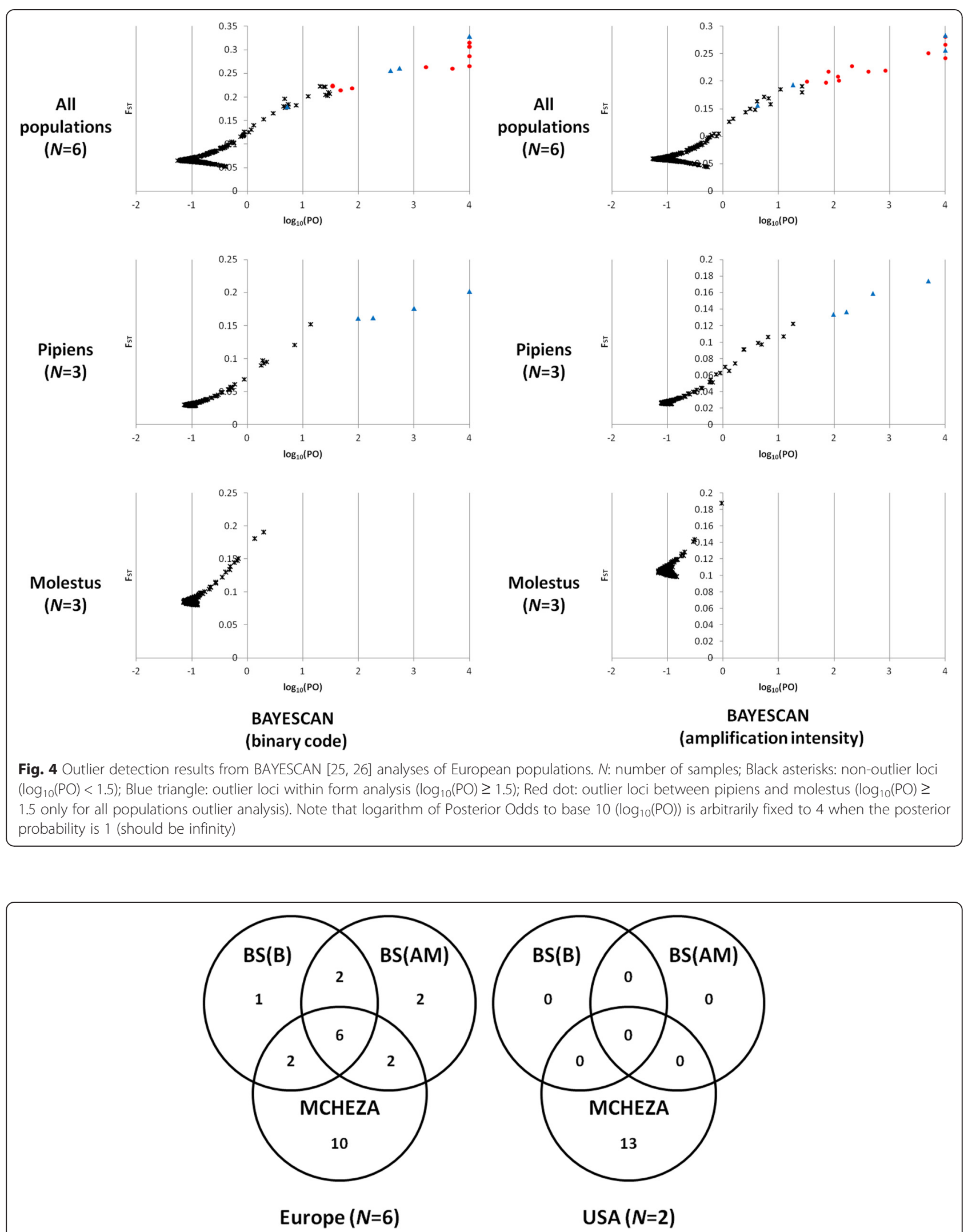

Fig. 5 Number of loci detected as outliers in Europe and USA by each method and replicated as outliers in multiple methods. BS(B): BAYESCAN with binary code [25]; BS(AM) BAYESCAN with amplification intensity matrix [26]; MCHEZA: MCHEZA with binary code [27]; N: number of samples 
populations. However, the molestus form presents ecological traits more adaptable to laboratory conditions (i.e., autogeny and stenogamy) when compared with the pipiens form, which theoretically could lead to higher differentiation in pipiens than molestus when the laboratory colonies were created. Therefore, the highest differentiation observed for the molestus sample of Chicago (USA) may have resulted from the additive effect of underground habitat colonization that isolated this population and the subsequent laboratory colony establishment/maintenance (at a colony density of $\approx 2000$ adult specimens, varying between 800 and 3200).

Multilocus screening by AFLP is able to identify candidate loci linked to adaptive genetic variation (i.e., outlier loci) that may be associated with mechanisms of selection and species adaptation [30]. The anonymous random information of AFLP does not allow determining the distribution of outlier loci in the genome but this technique is able to identify consistent signals among geographic populations and estimate the proportion of outlier loci in the genome. On average, the percentage of loci scored as outliers in incipient species comparisons varies between 5 and $10 \%$ (range: 0.4-24.5\%; Michel et al. [5]). Thus, outlier rates between pipiens and molestus appear to be relatively low with $3.1 \%$ (25 outliers in 810 loci) of loci outlying in comparisons among European samples and $1.6 \%$ (13 outliers in 810 loci) between USA samples, with the latter actually being $3.2 \%$ if it is considered that only half as many markers were scored in USA samples $(N=406)$.

Such outlier rates are comparable to those obtained using a SNP-chip (3.6 \% of outliers) to compare the genomes of the $\mathrm{M}$ and $\mathrm{S}$ molecular forms of the malaria vector Anopheles gambiae s.s. (now named as Anopheles coluzzii and Anopheles gambiae s.s. [31]), from GuineaBissau [32]. Interestingly, the proportion of outlier loci found in Guinea-Bissau, an area of exceptionally high hybridisation, was much lower than from inter-form comparisons in Ghana and Cameroon, where gene flow is much lower [32]. Similarity between the outlier rates found in the present analysis in Europe (Cx. pipiens s.s.) and Guinea-Bissau (An. gambiae s.s.) might be expected since both included the analysis of sympatric mosquito populations with elevated hybridization [13,33]. Estimates below the average have also been found in other sympatric populations of closely related insects with gene flow $[34,35]$.

The low genomic divergence in Europe between pipiens and molestus forms (outlier rates of $3.1 \%$ and an $F_{S T}$ average of 0.041) and the lower $A v \cdot q_{i}$ in Portuguese samples that indicate a higher background noise in pipiens than molestus samples are consistent with a pattern of asymmetric introgression from molestus into pipiens [36] previously observed in two distinct geographical areas (ca. $2700 \mathrm{~km}$ apart) of southern Europe with similar landscapes (Comporta and Thessaloniki [13, 16]). Gomes et al. [13] hypothesized that this pattern could be promoted by differences in mating strategies between the $C x$. pipiens s.s. forms: stenogamous molestus males (i.e., indoor opportunistic behaviour) and eurygamous pipiens males (i.e., outdoor swarm-based specialist behaviour). In fact, indoor mating has been associated with a breakdown of assortative mating between molecular forms of An. gambiae [37].

The similar outlier rates of USA and Europe inter-form analysis contrast with the higher $F_{S T}$ estimates found in USA inter-form comparisons $(\approx 1.9 \times$ higher on average) than European inter-form comparisons (Table 1). This low outlier rates in USA may be explained by form-specific signal lost in the molestus sample of Chicago (USA) due to founder effects and genetic drift in their colony establishment and maintenance, a phenomenon previously observed in Anopheles spp. laboratory colonies [38]. This pattern is also consistent with high intra/inter-form differentiation observed in colony and field collected molestus of Chicago [29] suggesting that underground colonization may have played a role in this divergence pattern.

When the two inter-form outlier analyses (European and USA) were compared, only two loci (0.25\%), Mix3D4_41 and Mix4D4_027, were found with a consistent outlier signal in both Europe and USA. These loci are likely to be associated with genomic regions involved in ecological speciation and/or in the adaptation to anthropogenic habitats by the molestus form. The capacity of molestus to occupy underground habitats associated with humans, such as subways, sewers and caves [11], has been promoted by stenogamy and autogeny, which allow a continuous existence in confined habitats with low availability of blood meal sources. These traits are retained even when the molestus form coexists with the pipiens form in aboveground habitats, such as in the case of Comporta, Portugal [13]. Likewise, there was a tendency for molestus individuals to occupy aboveground indoor habitats in this region [39]. In mosquitoes, habitat segregation has been considered a major factor underlying the divergence between the $\mathrm{M}$ and $\mathrm{S}$ forms of An. gambiae s.s. $[40,41]$. Ecological postmating barriers are expected to act against maladapted hybrids in the alternate $M$ versus $S$ larval habitats [42]. Moreover, autogeny and overwintering diapause are ecological traits essential to survive under non-ideal conditions (i.e., low host availability and low temperature) that may lead to energetic costs [43, 44]. These two ecological traits may play an important role in ecological postmating barriers acting against maladapted hybrids of $C x$. pipiens s.s. forms.

\section{Conclusion}

This study supports the status of the molestus and pipiens forms as distinct evolutionary entities with low 
genomic divergence that are likely to be in a process of incipient speciation. However, the anonymous information (i.e., lack of sequence) given by AFLP screening makes identification of genomic regions, genes, and mutations involved in the adaptation and speciation process difficult [30]. Further studies focusing on additional natural populations of $C x$. pipiens forms, using higher resolution genomic scans with high-throughput technologies are required in order to fully understand the genomic patterns in Cx. pipiens s.s. and identify processes that may be involved in the incipient speciation and habitat adaptation of pipiens and molestus forms.

\section{Methods}

\section{Mosquito samples}

Six field-collected samples from Europe were analysed; five from Portugal and one from the United Kingdom (UK). In addition, two USA samples were obtained from laboratory colonies (Table 2).

The USA form-specific colonies were established from mosquitoes collected in the area of Chicago, IL: molestus, by sampling a drainage sump using backpack aspirators and larval dipping in January 2009 [28]; and pipiens, from overwintering adults sampled by aspiration from a large culvert in January 2010. Both colonies were maintained by the methodology described in Mutebi and Savage [28]. Colonies were maintained at $27.5{ }^{\circ} \mathrm{C}$ and $80-90 \%$ relative humidity with light cycle of $14 \mathrm{~h}$ light and $10 \mathrm{~h}$ of darkness. Larvae were fed with a finely-ground mixture of $39.4 \%$ TetraMin flakes (Tetra Holdings, Blacksburg, VA), 51.7 \% liver powder (MP Biomedicals, Solon, $\mathrm{OH}$ ), and $8.9 \%$ brain/heart infusion (ICN Biomedicals, Aurora, $\mathrm{OH}$ ), and adult mosquitoes were offered $10 \%$ sucrose solution and raisins. Colonies were maintained separately in $45.7 \mathrm{~cm} \times 45.7 \mathrm{~cm}(18 \mathrm{in} \times 18$ in $)$ metal cages (BioQuip, Rancho Dominquez, CA) with approximately $2000(800-3200)$ adult specimens by the weekly addition of pupae in cups. The pipiens colony was also offered a bloodmeal once per week composed of defibrinated chicken or goose blood (Colorado Serum Company, Denver, $\mathrm{CO}$ ) using a Hemotek membrane feeding system (Discovery Workshop, Accrington, England). The mosquitoes used in the present study were taken from the colonies in February 2011.

In Portugal, indoor resting mosquito collections with mechanical aspirators were carried out in Comporta between May 2005 and August 2006 [13], in Alqueva (June 2007) and in Sandim (August 2010) (Table 2). A second mosquito collection in Comporta was performed outdoors, using CDC-light traps that were hung in trees, between July and August 2010 [39].

The indoor and outdoor collections carried out in Comporta were characterised with respect to their molestus and pipiens composition by the established microsatellite-based genetic backgrounds associated with particular bioecological traits, as described in previous publications [13, 39] (Table 2). The remaining samples of Alqueva and Sandim were provisionally identified as molestus by a diagnostic size polymorphism in the 5' flanking region of the CQ11 microsatellite (CQ11FL) [45]. This marker has proven to be useful to identify the presence of molestus and pipiens forms at the population level, but it is only partially effective in discriminating forms at the individual level $[13,16,39]$.

The sampling in the UK took place in March 2010, at the veterinary facility of the University of Liverpool, Leahurst, Wirral. Adults overwintering inside farm buildings (a typical behaviour of the pipiens form) were collected by Pyrethrum Spray Collection and were provisionally classified as pipiens by the CQ11FL marker (Table 2).

For all samples, DNA extraction from individual female mosquitoes was performed using the DNeasy blood and tissue kit (Qiagen, Inc., Manchester, UK).

Table 2 Localities of the samples used in the AFLP protocol

\begin{tabular}{|c|c|c|c|c|c|c|c|c|c|c|}
\hline Country & Locality & Latitude & Longitude & Year & Method & Form & Insectary & Code & $N$ & Ref \\
\hline \multirow[t]{5}{*}{ Portugal } & \multirow[t]{2}{*}{ Alqueva } & \multirow[t]{2}{*}{$38^{\circ} 17^{\prime} 54^{\prime \prime} \mathrm{N}$} & \multirow[t]{2}{*}{$7^{\circ} 35^{\prime} 17^{\prime \prime} \mathrm{W}$} & 2007 & $\mathbb{R}$ & molestus $^{a}$ & $\mathrm{Au} / \mathrm{St}$ & M_Al & 15 & - \\
\hline & & & & 2010 & CDC & pipiens & - & P_CC & 42 & [39] \\
\hline & \multirow{2}{*}{ Comporta } & \multirow{2}{*}{$38^{\circ} 21^{\prime} 09^{\prime \prime} \mathrm{N}$} & \multirow{2}{*}{$8^{\circ} 46^{\prime} 51 \mathrm{lW}$} & $2005-2006$ & \multirow{2}{*}{ IR } & molestus & $\mathrm{Au} / \mathrm{St}$ & M_CS & 50 & [13] \\
\hline & & & & $2005-2006$ & & pipiens & $\mathrm{N}-\mathrm{Au} / \mathrm{N}-\mathrm{St}$ & P_CS & 35 & [13] \\
\hline & Sandim & $41^{\circ} 01^{\prime} 19^{\prime \prime} \mathrm{N}$ & $8^{\circ} 30^{\prime} 20^{\prime \prime} \mathrm{W}$ & 2010 & $\mathbb{R}$ & molestus $^{a}$ & - & M_Sa & 39 & - \\
\hline UK & Wirral & $53^{\circ} 17^{\prime} 24^{\prime \prime} \mathrm{N}$ & $3^{\circ} 02^{\prime} 011^{\prime \prime} \mathrm{W}$ & 2010 & $|\mathrm{R}-|$ & pipiens $^{a}$ & - & P_Wi & 56 & - \\
\hline \multirow[t]{2}{*}{ USA } & Chicago & $41^{\circ} 43^{\prime} 09^{\prime \prime} \mathrm{N}$ & $87^{\circ} 45^{\prime} 23^{\prime \prime} \mathrm{W}$ & 2010 & MA & pipiens & $\mathrm{N}-\mathrm{Au} / \mathrm{N}-\mathrm{St}$ & P_Ch & 43 & - \\
\hline & & $41^{\circ} 39^{\prime} 49^{\prime \prime} \mathrm{N}$ & $87^{\circ} 36^{\prime} 30^{\prime \prime} \mathrm{W}$ & 2009 & BA/LC & molestus & $\mathrm{Au} / \mathrm{St}$ & M_Ch & 39 & [28] \\
\hline
\end{tabular}

Year: collection year and establishment of laboratory colony in USA. IR: Indoor resting collection with mechanical aspirators; IR-I: Indoor resting collections using insecticide spraying; CDC: outdoor collections performed by CDC light traps in trees; MA: collections using hand-held mechanical aspirators (Clarke, Roselle, IL, USA); BA: Collections by backpack aspirator (Model 1412; BioQuip, Rancho Dominguez, CA, USA); LC: larvae collections using dippers. Form: identification based in a combination of molecular analysis and ecological data; ${ }^{a}$ specimens provisionally identified by the CQ11FL marker. Insectary: insectary experiments performed to determine autogeny and stenogamy [13, 28]; Au: autogenous; N-Au: non-autogenous; St: stenogamous; N-St: non-stenogamous. N: sample size. Ref: References 


\section{AFLP protocol}

The DNA concentration of each sample was fluorometrically quantified by the Quant-i $\mathrm{T}^{\mathrm{TN}}$ PicoGreen $^{\circ}$ dsDNA reagent and kit (Invitrogen ${ }^{\mathrm{Tm}}$, Paisley, UK) as recommended by Wilding et al. [46].

For each specimen, $100 \mathrm{ng}$ of genomic DNA was used as template in the AFLP protocol described by Wilding et al. [47], but without a dilution step between the ligation and the pre-selective PCRs. Primers used in the amplification are provided in Additional file 1: Table S1. Selective primers were labelled to allow separation of amplified products on a CEQ ${ }^{\mathrm{TM}} 8000$ capillary sequencer (Beckman Coulter Inc., CA, USA) using the Beckman 600 DNA size standard kit - to quantify fragments between 50 and 700 base pairs. Peaks were only called if they exceeded thresholds of both $3 \%$ of the maximum fluorescence peak height and 500 relative fluorescence units of intensity. A raw matrix of the marker peak data was defined using a bin width of $1 \mathrm{bp}$. These conditions were selected because they showed the highest proportion of reproducible peaks during optimization.

Special precautions were taken in order to avoid misinterpretation due to peak size homoplasy (i.e., lack of homology of co-migrating fragments) [20] and genotyping errors. Automated scoring and replicated samples were used to increase the objectivity of the genotyping process.

The approach of Whitlock et al. [19] was implemented to determine which peaks from the raw data matrix could be scored reliably. A two-step approach, performed by AFLPscore [19], was used to score the peaks from the raw data matrix, with a first step in which the relative threshold in the fluorescence peak height was set at $20 \%$ in order to select the loci from the raw matrix, and a second at $15 \%$ to score the chosen loci. AFLP analysis was repeated on a sub-set of samples for all the primer combinations (Additional file 1: Table S2) to assess technical error using both mismatch rates and Bayesian AFLPscore error analysis (proportion of mismatches; probability of mis-scoring allele 1 as allele 0 , denoted E1; and probability of mis-scoring allele 0 as allele 1, denoted E2) [19].

The number of loci per primer combination and proportion of loci at four fragment size groups $(<125 \mathrm{bp}$; 125-199 bp; 200-299 bp; >299 bp) were determined in order to infer the effects of peak size homoplasy (i.e., lack of homology of co-migrating fragments) in the data set. This phenomenon is one of the major technical challenges in the AFLP technique and may cause overestimation of allele frequencies or reduction in performance for detection of loci under selection. A balanced data set avoiding a high proportion of loci with low fragment size $(<125 \mathrm{bp})$ and high number of loci per combination ( $>100$ loci) is recommended to minimise homoplasy in AFLP data sets [20].

\section{Population genetic structure and genetic diversity}

Bayesian clustering analysis as implemented by STRUCTURE 2.3.3 [21] was used to infer population substructure/ancestry from the AFLP data set without prior information of sampling groups, under conditions of admixture ( $\alpha$ allowed to vary between 0 and 10) with allele frequencies correlated among populations $(\lambda$ set at the default value of 1 ). Ten independent runs, with $10^{5}$ iterations during burn-in followed by $20^{5}$ replications, were performed for each value of $K(K=1$ to 10 clusters for all samples). Information from the output of each $K$ (10 runs) was compiled by the Greedy method implemented in CLUMPP [48]. To infer the most likely number of clusters in the sample, two ad hoc approaches were implemented by structure harvester v.0.6.94 [49]: i) an estimation of $\ln [\operatorname{Pr}(\mathrm{X} \mid K)]$ [21], and ii) the $\Delta K$ statistic [50]. Average values of probability of membership per sample $\left(A v \cdot q_{i}\right)$ were determined to infer the degree of admixture in each sample.

Divergence among the sampled populations was assessed by analysis of molecular variance (AMOVA [23]) using GENALEX 6.41 [22].

Principal Coordinates Analysis was used to visualise patterns of genetic differentiation among samples in two-dimensional plots. Calculations were performed in GENALEX 6.41 [22] using the standardised covariance method for the distance matrix conversion.

Pairwise estimates of $F_{S T}$ between collection sites were calculated in AFLP-SURV [24]. To construct a bootstrapped neighbour-joining tree, 10,000 random replicates of pairwise $F_{S T}$ tables (based on all loci) were calculated also in AFLP-SURV. These tables were used as input for PHYLIP 3.68 [51], in which the programs NEIGHBOR and CONSENSE were used to produce the bootstrapped tree. Figtree v.1.3.1 [52] was used to visualize the tree.

The number of polymorphic loci, proportion of polymorphic loci at the $5 \%$ level, and expected heterozygosity [53] were estimated assuming Hardy-Weinberg equilibrium in AFLP-SURV. Chi-squared tests on contingency tables - available in VassarStats [54] - were performed to assess differences between pipiens and molestus forms for these genetic diversity estimates. To perform a paired chisquare analysis, diversity estimates were averaged between the pipiens samples from Comporta (CDC light traps) and Wirral and compared to the mean of the molestus samples from Alqueva and Sandim.

\section{Loci divergence and outlier loci detection}

BAYESCAN 2.1 [25] was used to compare neutral models with models including selection and to estimate the posterior odds (PO) in support of selection over neutrality for each locus. BAYESCAN was applied to the binary code (i.e., allele presence/absence) typical for 
dominant markers. A second approach was implemented using the amplification intensity matrix which can provide additional information from AFLP marker data and yield power similar to that of co-dominant markers [26]. We conducted 20 pilot runs with a length of 5000 iterations each followed by an additional burn-in of 50,000 iterations; preceding tests indicated that this was sufficient to achieve convergence in the Markov chain Monte Carlo. Default values were used for sample size (5000) and thinning interval (10). The prior odds were set as 10 as recommended by the manual for data with a few hundred loci. For the amplification intensity matrix we used 0.10 as threshold for the recessive genotype as a fraction of maximum band intensity. Outliers were identified by the direct estimation of a posterior probability for each locus using a reversible-jump Monte Carlo Markov chain (threshold: $\log _{10}(\mathrm{PO})>1.5$ ).

The third approach for outlier detection used the DFDIST algorithm [55], as implemented in the software MCHEZA [27]. The DFDIST method compares empirical $F_{S T}$ values to a null distribution derived from coalescent simulations and determines the probability that observed $F_{S T}$ values are as large as, or larger than, the observation under neutrality. Runs were conducted under 'neutral mean $F_{S T}$ ', which involves computing the initial mean $F_{S T}$ uninfluenced by outliers, with the following default settings: 50,000 simulations; 0.1 false discovery rate; 0.1 theta; 0.25 beta-a; and 0.25 beta-b. The significance threshold for outlier detection was set at $\geq 0.95$ percentile of simulations.

Detection of outlier loci was conducted differently according to the geographic origin of samples. For European samples, outliers were first identified over all six samples and then within molestus and pipiens samples. Outliers identified among all populations, but not among either of the within-form analyses, were considered as candidate loci under divergent selection between pipiens and molestus. This indirect approach could not be applied to the USA samples because only one sample from each form was analysed. Therefore, outliers were identified from the direct comparison between pipiens and molestus samples. The direct approach between two population samples requires a cautious interpretation because outlier detection methods are known to be less robust with a small number of populations for comparison [25].

Pairwise analyses among all populations were performed by MCHEZA in order to map divergence across the $F_{S T}$ values distribution (i.e., minimum value, mean, median, maximum value and percentiles).

\section{Additional file}

Additional file 1: Table S1. Primers used in the AFLP protocol. Table S2 Error rates of the loci obtained by each primer combination in the selective amplification. Table S3. Population diversity of the eight populations used in the study. Table S4. Divergence estimates based on FST pairwise sample analysis per locus within pipiens and molestus samples. Table S5. Divergence estimates based in FST pairwise sample analysis per locus between pipiens and molestus samples. Table S6. Loci detected as outliers in each comparative analysis (Europe and USA). Table S7. Proportion of the loci by fragment size in the Overall and USA data. Fig. S1. Graphics of ad hoc approaches to infer the number of clusters ( $K$ ) in STRUCTURE analysis with all samples. Fig. S2. Outlier detection results from MCHEZA analyses. (PDF $451 \mathrm{~kb}$ )

\section{Competing interests}

The authors declare that they have no competing interests.

\section{Authors' contributions}

BG, CAS, MTN, HMS and APGA carried out sample collections and provided samples from insectary. Molecular analyses were conducted by BG and CSW. $B G, C S W, D W$ and MJD performed the genetic data analysis. BG, CSW, JP and MJD conceived the study and designed the experiments. BG, JP and MJD drafted the manuscript with the contributions of CSW, DW. All authors read and approved the final manuscript.

\section{Acknowledgments}

We thank Tiago Antão for the technical support given to the data analysis. This study was funded by Fundação para a Ciência e a Tecnologia/, Portugal (POCI/BIA-BDE/57650/2004 and PPCDT/BIA-BDE/57650/2004). Bruno Gomes was funded by a PhD fellowship of Fundação para a Ciência e Tecnologia/ MCTES (SFRH/BD/36410/2007).

\section{Author details}

Global Health \& Tropical Medicine, Instituto de Higiene e Medicina Tropical, Universidade Nova de Lisboa, Rua da Junqueira 100, 1349-008 Lisbon, Portugal. ${ }^{2}$ Department of Vector Biology, Liverpool School of Tropical Medicine, Pembroke Place, Liverpool L3 5QA, UK. ${ }^{3}$ School of Natural Sciences and Psychology, Liverpool John Moores University, Liverpool L3 3AF, UK. ${ }^{4}$ Centers for Disease Control and Prevention, 3156 Rampart Road, Fort Collins, CO 80521, USA.

Received: 1 June 2015 Accepted: 3 September 2015

Published online: 16 September 2015

\section{References}

1. Hopkins R, Rausher MD. Identification of two genes causing reinforcement in the Texas wildflower Phlox drummondii. Nature. 2011;469:411-4.

2. Nosil P. Degree of sympatry affects reinforcement in Drosophila. Evolution. 2012;67:868-72.

3. Nosil P, Harmon $\sqcup$, Seehausen O. Ecological explanations for (incomplete) speciation. Trends Ecol Evol. 2009;24:145-56.

4. Wu C-I. The genic view of the process of speciation. J Evol Biol. 2001;14:851-65.

5. Michel AP, Sim S, Powell THQ, Taylor MS, Nosil P, Feder JL. Widespread genomic divergence during sympatric speciation. Proc Natl Acad Sci U S A. 2010;107:9724-9

6. Clarkson CS, Weetman D, Essandoh J, Yawson AE, Maslen G, Manske M, et al. Adaptive introgression between Anopheles sibling species eliminates a major genomic island but not reproductive isolation. Nat Commun. 2014:5:4248

7. Soria-Carrasco V, Gompert Z, Comeault AA, Farkas TE, Parchman TL, Johnston JS, et al. Stick insect genomes reveal natural selection's role in parallel speciation. Science. 2014;344:738-42.

8. Hubálek Z. Mosquito-borne viruses in Europe. Parasitol Res. 2008;103:29-43.

9. Harbach RE, Harrison BA, Gad AM. Culex (Culex) molestus Forskål (Diptera, (ulicidae) - neotype designation, description, variation, and taxonomic status. Proc Entomol Soc Wash. 1984;86:521-42.

10. Harbach R, Dahl C, White G. Culex (Culex) pipiens Linnaeus (Diptera, Culicidae) - concepts, type designations, and description. Proc Entomol Soc Wash. 1985;87:1-24.

11. Vinogradova EB. Culex pipiens pipiens mosquitoes: taxonomy, distribution, ecology, physiology, genetics, applied importance and control. Sofia: Pensoft; 2000 
12. Fonseca DM, Keyghobadi N, Malcolm CA, Mehmet C, Schaffner F, Mogi M, et al. Emerging vectors in the Culex pipiens complex. Science. 2004;303:1535-8

13. Gomes B, Sousa CA, Novo MT, Freitas FB, Alves R, Corte-Real AR, et al. Asymmetric introgression between sympatric molestus and pipiens forms of Culex pipiens (Diptera: Culicidae) in the Comporta region, Portugal. BMC Evol Biol. 2009;9:262

14. Callot J, Van Ty D. Sur quelques souches françaises de Culex pipiens L. Bull Soc Pathol Exot Filiales. 1943;36:229-32.

15. Pasteur N, Rioux JA, Guilvard E, Pech-Perieres J. Nouvelle mention pour le "Midi" méditerranéen, de populations naturelles anautogènes. Ann Parasitol Hum Comp. 1977;11:187-93.

16. Gomes B, Kioulos E, Papa A, Almeida APG, Vontas J, Pinto J. Distribution and hybridization of Culex pipiens forms in Greece during the West Nile virus outbreak of 2010. Infect Genet Evol. 2013;16:218-25.

17. Rizzoli A, Bolzoni L, Chadwick EA, Capelli G, Montarsi F, Grisenti M, et al. Understanding West Nile virus ecology in Europe: Culex pipiens host feeding preference in a hotspot of virus emergence. Parasit Vectors. 2015;8:213.

18. Nelms BM, Kothera L, Thiemann T, Macedo PA, Savage HM, Reisen WK Phenotypic variation among Culex pipiens complex (Diptera: Culicidae) populations from the Sacramento Valley, California: horizontal and vertical transmission of West Nile virus, diapause potential, autogeny, and host selection. Am J Trop Med Hyg. 2013:89:1168-78.

19. Whitlock R, Hipperson H, Mannarelli M, Butlin RK, Burke T. An objective, rapid and reproducible method for scoring AFLP peak-height data that minimizes genotyping error. Mol Ecol Resour. 2008;8:725-35.

20. Caballero A, Quesada H, Rolán-Alvarez E. Impact of amplified fragment length polymorphism size homoplasy on the estimation of population genetic diversity and the detection of selective loci. Genetics. 2008;179:539-54.

21. Pritchard JK, Stephens M, Donnelly P. Inference of population structure using multilocus genotype data. Genetics. 2000;155:945-59.

22. Peakall R, Smouse PE. Genalex 6: genetic analysis in Excel. Population genetic software for teaching and research. Mol Ecol Notes. 2006;6:288-95.

23. Excoffier L, Smouse PE, Quattro JM. Analysis of molecular variance inferred from metric distances among DNA haplotypes: application to human mitochondrial DNA restriction data. Genetics. 1992;131:479-91.

24. Vekemans $X$, Beauwens T, Lemaire M, Roldán-Ruiz I. Data from amplified fragment length polymorphism (AFLP) markers show indication of size homoplasy and of a relationship between degree of homoplasy and fragment size. Mol Ecol. 2002:11:139-51.

25. Foll M, Gaggiotti O. A genome-scan method to identify selected loci appropriate for both dominant and codominant markers: a bayesian perspective. Genetics. 2008;180:977-93.

26. Fischer MC, Foll M, Excoffier L, Heckel G. Enhanced AFLP genome scans detect local adaptation in high-altitude populations of a small rodent (Microtus arvalis). Mol Ecol. 2011;20:1450-62

27. Antao T, Beaumont MA. Mcheza: a workbench to detect selection using dominant markers. Bioinformatics. 2011;27:1717-8.

28. Mutebi J-P, Savage HM. Discovery of Culex pipiens pipiens form molestus in Chicago. J Am Mosq Control Assoc. 2009;25:500-3.

29. Kothera L, Godsey M, Mutebi J-P, Savage HM. A comparison of aboveground and belowground populations of Culex pipiens (Diptera: Culicidae) mosquitoes in Chicago, Illinois, and New York City, New York, using microsatellites. J Med Entomol. 2010;47:805-13.

30. Paris M, Meyer C-L, Blassiau C, Coissac E, Taberlet P, Després L. Two methods to easily obtain nucleotide sequences from AFLP loci of interest. Methods Mol Biol. 2012;888:91-108.

31. Coetzee M, Hunt RH, Wilkerson R, Della Torre A, Coulibaly MB, Besansky NJ. Anopheles coluzzii and Anopheles amharicus, new members of the Anopheles gambiae complex. Zootaxa. 2013;3619:246-74.

32. Weetman D, Wilding CS, Steen K, Pinto J, Donnelly MJ. Gene flow-dependent genomic divergence between Anopheles gambiae $\mathrm{M}$ and $\mathrm{S}$ forms. Mol Biol Evol. 2012;29:279-91.

33. Oliveira E, Salqueiro P, Palsson $K$, Vicente $J L$, Arez AP, Jaenson TG, et al. High levels of hybridization between molecular forms of Anopheles gambiae from Guinea Bissau. J Med Entomol. 2009:45:1057-63.

34. Gompert Z, Lucas LK, Nice CC, Fordyce JA, Forister ML, Buerkle CA. Genomic regions with a history of divergent selection affect fitness of hybrids between two butterfly species. Evolution. 2012;66:2167-81.
35. Nadeau NJ, Whibley A, Jones RT, Davey JW, Dasmahapatra KK, Baxter SW, et al. Genomic islands of divergence in hybridizing Heliconius butterflies identified by large-scale targeted sequencing. Philos Trans R Soc Lond B Biol Sci. 2012:367:343-53.

36. Guichoux E, Garnier-Géré P, Lagache L, Lang T, Boury C, Petit RJ. Outlier loci highlight the direction of introgression in oaks. Mol Ecol. 2013;22:450-62.

37. Dao A, Adamou A, Yaro AS, Maïga HM, Kassogue Y, Traoré SF, et al. Assessment of alternative mating strategies in Anopheles gambiae: Does mating occur indoors? J Med Entomol. 2008;45:643-52.

38. Norris DE, Shurtleff AC, Touré YT, Lanzaro GC. Microsatellite DNA polymorphism and heterozygosity among field and laboratory populations of Anopheles gambiae s.s. (Diptera: Culicidae). J Med Entomol. 2001;38:336-40.

39. Gomes B, Sousa CA, Vicente JL, Pinho L, Calderón I, Arez E, et al. Feeding patterns of molestus and pipiens forms of Culex pipiens (Diptera: Culicidae) in a region of high hybridization. Parasit Vectors. 2013;6:93.

40. Diabaté A, Dabiré RK, Heidenberger K, Crawford J, Lamp WO, Culler LE, et al. Evidence for divergent selection between the molecular forms of Anopheles gambiae: role of predation. BMC Evol Biol. 2008;8:5

41. Diabaté A, Dao A, Yaro AS, Adamou A, Gonzalez R, Manoukis NC, et al. Spatial swarm segregation and reproductive isolation between the molecular forms of Anopheles gambiae. Proc R Soc Ser B Biol Sci. 2009:276:4215-22.

42. Turner TL, Hahn MW. Genomic islands of speciation or genomic islands and speciation? Mol Ecol. 2010;19:848-50.

43. Lankinen P, Tyukmaeva VI, Hoikkala A. Northern Drosophila montana flies show variation both within and between cline populations in the critical day length evoking reproductive diapause. J Insect Physiol. 2013;59:745-51.

44. Kassim NFA, Webb CE, Russell RC. Is the expression of autogeny by Culex molestus Forskal (Diptera: Culicidae) influenced by larval nutrition or by adult mating, sugar feeding, or blood feeding? J Vector Ecol. 2012;37:162-71.

45. Bahnck CM, Fonseca DM. Rapid assay to identify the two genetic forms of Culex (Culex) pipiens L. (Diptera: Culicidae) and hybrid populations. Am J Trop Med Hyg. 2006;75:251-5.

46. Wilding CS, Weetman D, Steen K, Donnelly MJ. Accurate determination of DNA yield from individual mosquitoes for population genomic applications. Insect Sci. 2009;16:361-3.

47. Wilding CS, Butlin RK, Grahame J. Differential gene exchange between parapatric morphs of Littorina saxatilis detected using AFLP markers. J Evol Biol. 2001;14:611-9.

48. Jakobsson M, Rosenberg NA. CLUMPP: a cluster matching and permutation program for dealing with label switching and multimodality in analysis of population structure. Bioinformatics. 2007;23:1801-6.

49. Earl DA, VonHoldt BM. Structure Harvester: a website and program for visualizing Structure output and implementing the Evanno method. Conserv Genet Resour. 2012;4:359-61.

50. Evanno G, Regnaut S, Goudet J. Detecting the number of clusters of individuals using the software Structure: a simulation study. Mol Ecol. 2005:14:2611-20.

51. Felsenstein J. PHYLIP (phylogeny inference package). Seattle, Washington, USA: Version 3.68. Department of Genome Sciences, University of Washington; 2004

52. Rambaut A. FigTree. Edinburgh, UK: Version 1.3.1. University of Edinburgh; 2009.

53. Lynch M, Milligan BG. Analysis of population genetic-structure with RAPD markers. Mol Ecol. 1994;3:91-9.

54. Lowry R. VassarStats: web site for statistical computation. 2013 http://vassarstats.net/index.html.

55. Beaumont MA, Balding DJ. Identifying adaptive genetic divergence among populations from genome scans. Mol Ecol. 2004;13:969-80. 